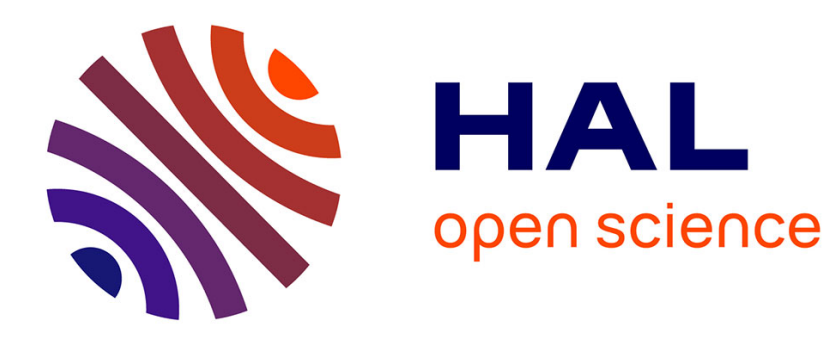

\title{
Le grignotage des forêts dans la Lorraine industrielle
} Jean-Claude Bonnefont

\section{To cite this version:}

Jean-Claude Bonnefont. Le grignotage des forêts dans la Lorraine industrielle. Revue forestière française, 1979, 31 (1), pp.63-72. 10.4267/2042/21958 . hal-03397164

\section{HAL Id: hal-03397164 \\ https://hal.science/hal-03397164}

Submitted on 22 Oct 2021

HAL is a multi-disciplinary open access archive for the deposit and dissemination of scientific research documents, whether they are published or not. The documents may come from teaching and research institutions in France or abroad, or from public or private research centers.
L'archive ouverte pluridisciplinaire HAL, est destinée au dépôt et à la diffusion de documents scientifiques de niveau recherche, publiés ou non, émanant des établissements d'enseignement et de recherche français ou étrangers, des laboratoires publics ou privés. 


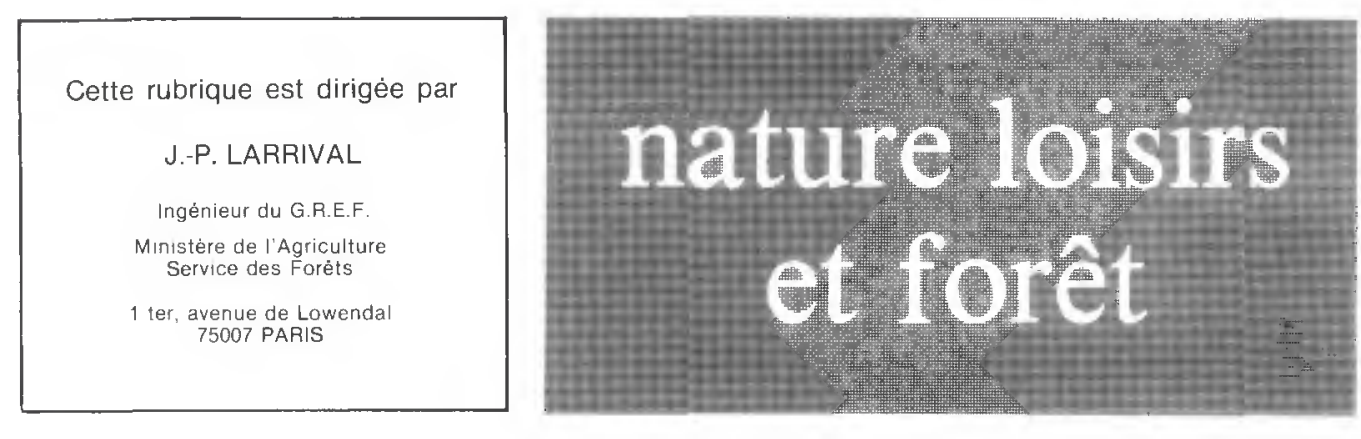

\section{LE GRIGNOTAGE DES FORÊTS DANS LA LORRAINE INDUSTRIELLE}

La Lorraine est une des régions de France les plus boisées : les torêts occupent $36 \%$ de son territoire. Mais l'originalité profonde de cette région, si on la compare par exemple au nord de la France, est que même les pays d'industrie extractive ou d'industrie sidérurgique y possèdent des taux de boisement élevés. II nous a semblé intéressant de poser ici le problème de la coexistence des torêts avec l'industrie lourde : coexistence de plus en plus difficile, car l'industrie a besoin de plus en plus d'espace, attire une population de plus en plus nombreuse, crée des nuisances de plus en plus graves. II en résulte un incessant grignotage dont nous nous proposons de décrire ici, à l'aide de quelques exemples, les mécanismes et les effets.

\section{APERÇU HISTORIQUE}

On est frappé, en regardant de près les statistiques, par l'importance des superficies boisées dans certaines parties de la Lorraine industrielle et minière : $51 \%$ du sol dans le canton de Moyeuvre-Grande, $68 \%$ dans la commune de Briey, $39 \%$ dans le canton de Forbach-Ville, $37 \%$ dans celui de Saint-Avold, etc. Partout ou presque, l'usine est associée à la forêt. Cette situation représente l'héritage d'un passé déjà lointain et traduit largement l'influence de la nature du sol. Les forêts représentent en Lorraine le négatit des grands détrichements agricoles d'autrefois. Ce sont des raisons surtout pédologiques qui expliquent la conservation des forêts soit sur les calcaires bajociens très résistants du Pays-Haut, soit sur les grès chimiquement très pauvres de la Warndt. Dès le Moyen-Age, la distinction globale entre les principaux massifs boisés et les territoires défrichés s'était déjà opérée. 


\section{J.C. BONNEFONT}

Par la suite, la présence de forêts susceptibles de fournir du bois de chauffage a certainement favorisé l'implantation d'industries, notamment de forges, de verreries, de salines. Mais l'industrie se montrait une bien moins grande consommatrice de terres que l'agriculture. Certes, elle a souvent conduit au pillage et à la dévastation de certaines forêts. Les archives contiennent de nombreuses plaintes contre les abus des concessionnaires des salines ou des maîtres de forges (1). De nombreuses forêts étaient réduites à l'état de taillis fort maigres, exploités avec une très courte révolution. Mais il ne pouvait pas être question de changer la destination forestière du sol, puisque l'implantation de l'usine elle-même dépendait de la survie des forêts fournissant le combustible autant que des gisements de matières premières. Si des déboisements à usage industriel ont eu lieu, ce fut surtout lors de la création de nouvelles verreries: autour de chaque verrerie se constituèrent des villages avec leur clairière de défrichement, aménagée à des fins agricoles et pastorales. Dans la Warndt, les créations de verreries se placent à la charnière des $X V l^{e}$ et $X V I^{e}$ siècles, sur les sites de Merlebach (1590), Freyming (1602), Creutzwald (1618), Vieille Verrerie (1618), puis plus tardivement encore au XVIII ${ }^{e}$ siècle à La Houve (1705) et Verrerie Sophie près de Forbach (1721).

Si on compare la carte de Cassini, réalisée au milieu du XVIII ${ }^{e}$ siècle avec la situation des annèes 1920, on est frappé par la faiblesse des modifications de limites forestières. L'action conservatrice de l'administration forestière se manifeste partout. Aussi bien dans le bassin ferrifère de Briey que dans le bassin houiller de Forbach, Ies essartements sont rares; quelques déboisements sont liés au passage de voies ferrées; mais les plus importants, dans la Warndt, sont des défrichements de caractère agricole, dûs à la création de fermes nouvelles, comme R. Capot-Rey l'a très bien montré dans sa thèse.

Ces observations sont confirmées pour le bassin sidérurgique de Briey-Thionville par une étude statistique reproduite dans "L'Est Républicain" (édition de Briey) du 9 juin 1931. Elle prouve que le développement de l'industrie sidérurgique entre 1878 et 1913 a consommé beaucoup plus de terrains à vocation agricole que de territoires forestiers.

\section{Tableau $N^{\circ} 1 .-$ Diminution des superticies cultivées et boisées de 1878 à 1913} dans les régions sidérurgiques.

\begin{tabular}{|c|c|c|}
\hline Unités géographiques & Terres cultivabies & Forêts \\
\hline Plateau d'Aumetz........ & 230 ha & 10 ha \\
\hline Vallée de la Fensch.... & 362 ha & 60 ha \\
\hline Vallée de l'Orne......... & 396 ha & - \\
\hline Vallée de la Moselle ............ & 630 ha & - \\
\hline Région de Thionville ............ & 242 ha & 65 ha \\
\hline
\end{tabular}

Cette situation s'explique par le fait que les usines sidérurgiques étaient implantées dans les vallées, depuis longtemps déboisées. Certes, les mines se trouvaient parfois établies au milieu des forêts du plateau, mais leur emprise au sol restait généralement très restreinte en raison de la modicité des installations de surface nécessaires à leur fonctionnement. Du moment que l'exode rural et l'abandon de certaines cultures comme la vigne libéraient d'importantes superficies de terres agricoles, il n'a pas été nécessaire de prélever aux dépens des forêts, les terrains dont on avait besoin pour étendre les usines et les cités.

(1) Dans la première moitiè du XVII|e siècle, les Wendel sont en procès avec les habitants des environs d'Hayange et un arrêt de la Cour de Bar finit par limiter leur exploitation á 600 arpents de bois par an. En 1758. Charles de Wendel déplace set installations en rachetant les forges de Sainte-Fontaine, dans la Warndt, oú les mêmes difficultés renaissent aprés quelques années. 


\section{L'ACCÉLÉRATION DU GRIGNOTAGE ET SES CAUSES}

Depuis les années 1920, les déboisements qui ont été pratiqués dans la Lorraine industrielle l'ont été, soit pour les besoins d'extension des usines, soit pour le logement de la maind'œuvre industrielle. Les données chiffrées manquent pour établir un bilan complet, mais l'exem. ple de la forêt de Saint-Avold peut permettre de se faire une idée précise de cette évolution. Cette forêt couvrait en 1928 une superficie de 3918 ha. De 1928 à 1974, les déboisements ont porté sur le tiers de cette surface, soit 1297 ha. Leur bilan s'établit de la façon suivante (²) :

- $9 \%$ seulement ont été rendus nécessaires par l'exploitation de la houille;

- $12 \%$ ont servi à aménager des cités ouvrières (Arcadia, Jeanne-d'Arc) pour lesquelles on aurait pu choisir d'autres sites;

\section{LA FORÊT DE SAINT-AVOLD}

\section{AUX ABORDS DU COMPLEXE INDUSTRIEL DE CARLING}

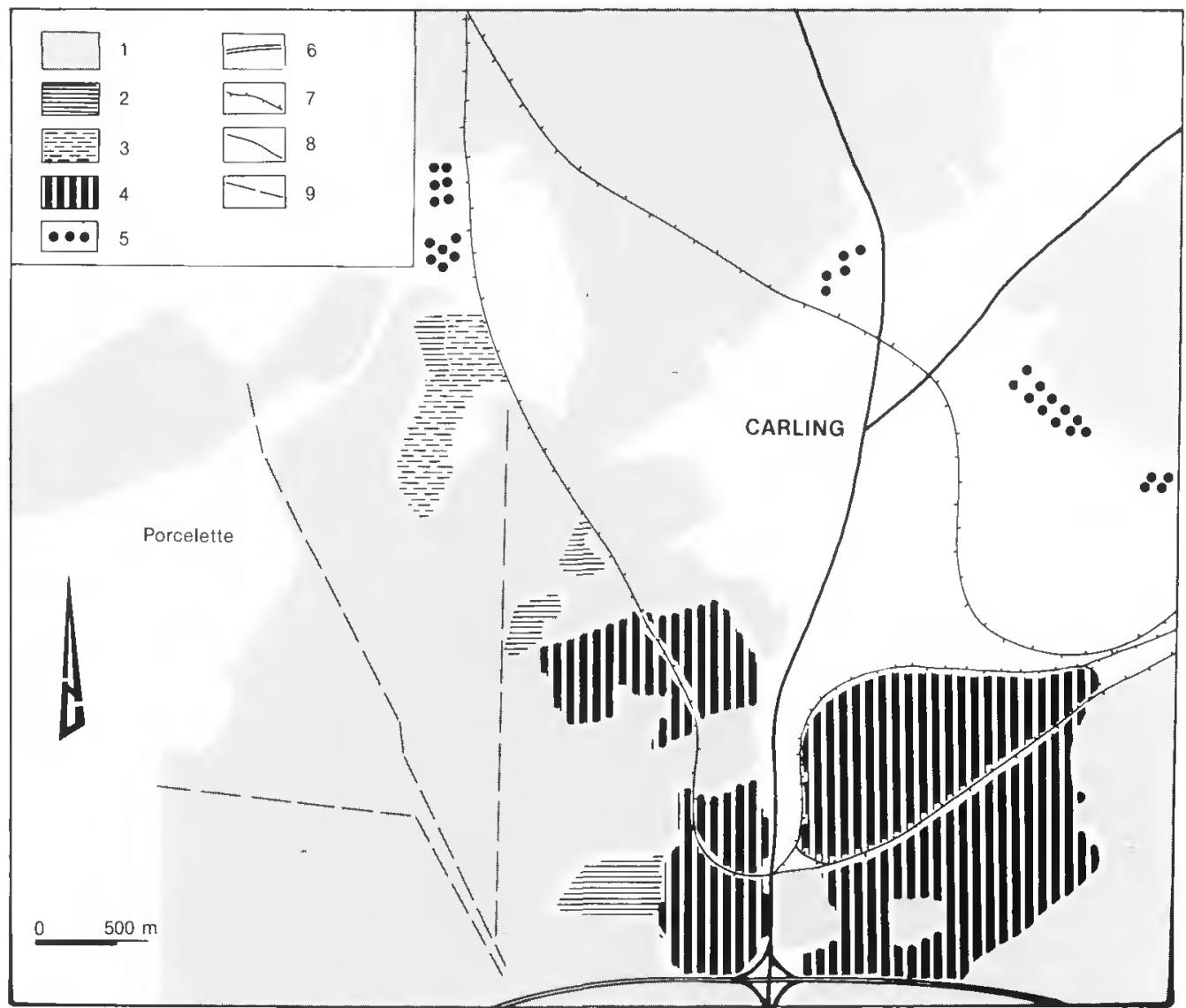

1 - forêt ; 2 - étang de décharge ; 3 - décharge de morts terrains ; 4-- déboisement industriel ; 5 - cité ouvrière en bordure de la forêt $; 6$ - autoroute; 7 -voie ferrèe ; 8 - route $; 9$-ligne électrique.

(2) D'après le procès-verbal de la réunion-tournée forestière du 17 octobre 1974 en forêt de Saint-Avold. 
- $42 \%$ ont permis de dégager l'emplacement nécessaire à la création de la centrale thermique Emile-Huchet et de la plate-torme d'industrie chimique de Carling. Déjà situées en bordure de la forêt domaniale de Saint-Avold, les installations des Houillères du bassin de Lorraine ne pouvaient pratiquement plus s'étendre qu'au détriment de celle-ci. L'administration ayant accepté l'échange d'une partie de la surface boisée contre d'autres terrains à vocation forestière, c'est un bois de pins qui a été sacrifié ;

- $37 \%$ enfin ont été pris atin de permettre le passage de lignes électriques, de conduites d'eau et de gaz ou encore de voies de circulation. Les servitudes sont en effet considérables en milieu industriel : l'alimentation en bas produits (schlamms, charbons pulvérulents) de la cokerie de Carling se fait à partir du lavoir de Freyming par un pipe-line long de $9 \mathrm{~km}$. Dautres conduites envoient vers les consommateurs le gaz produit sur place. De plus, la construction récente de l'autoroute A 32 a consommé beaucoup d'espace : elle traverse l'ensemble boisé selon son plus grand axe et c'est au milieu de la torêt de Saint-Avold que l'on a choisi d'établir divers équipements annexes (péage, base d'entretien, aires de services et de restauration). Enfin, la création au cœur de la forêt d'une station électrique a entraîné un fâcheux compartimentage de celle-ci par de grandes trouées linéaires. En raison de ces diverses lignes déboisées, on peut considérer que 230 ha environ de la forêt domaniale sont perturbés par les effets de lisière. En canalisant les gaz nocifs, ces couloirs favorisent la dégradation de la végétation sur une profondeur qui peut atteindre $25 \mathrm{~m}$.

Tableau N². - Réduction de superficie de la forêt de Saint-Avold (Moselle) de 1928 à 1974.

\begin{tabular}{|c|c|c|}
\hline FORÊT DÉTRUITE & $\begin{array}{l}\text { Voies de communication et transport d'énergie } \\
\text { Emprise des usines } \ldots \ldots \ldots \ldots \ldots \ldots \ldots \ldots \ldots \ldots \ldots \ldots \ldots \ldots \ldots \ldots \ldots \\
\text { Cités d'habitation } \ldots \ldots \ldots \ldots \ldots \ldots \ldots \ldots \ldots \ldots \\
\text { Extraction du charbon. } \ldots \ldots \ldots \ldots \ldots \ldots\end{array}$ & $\begin{array}{l}478 \text { ha } \\
548 \text { ha } \\
152 \text { ha } \\
119 \text { ha }\end{array}$ \\
\hline FORÊT SURVIVANTE : 2621 ha & $\begin{array}{l}\text { Souffrant d'effets de lisière } \ldots \ldots \ldots \ldots \ldots \ldots \ldots \\
\text { Atteinte par des effets écologiques indirects ... } \\
\text { Apparemment intacte } \ldots \ldots \ldots \ldots \ldots \ldots \ldots \ldots \ldots\end{array}$ & $\begin{array}{r}230 \text { ha } \\
570 \text { ha } \\
1821 \text { ha }\end{array}$ \\
\hline
\end{tabular}

Ce bilan établi en 1974 a été aggravé depuis cette date par de nouveaux déboisements. Dans le cadre d'une politique de modernisation de leurs installations et de réhabilitation du charbon lorrain, les Houillères du Bassin de Lorraine ont entrepris d'édifier un sixième groupe très puissant à la Centrale Emile-Huchet. L'aménagement de la plate-forme a entraîné le sacrifice d'une dizaine d'hectares supplémentaires à l'automne 1977. A ces déboisements directs s'ajoutent enfin près de Carling les dégâts dus à la pollution, notamment par le $\mathrm{SO}_{2}$. Dans la parcelle $n^{\circ} 151$, conservée en l'état à titre de témoin par l'Office national des forêts, tous les épicéas sont morts et dressent vainement vers le ciel leurs squelettes blanchis, tandis que les teuillus souffrent d'un dessèchement de la cime.

L'examen des photographies aériennes permet d'affirmer que l'exemple de la forêt de SaintAvold n'est pas un cas isolé. Dans tout le bassin houiller sarrois, de part et d'autre de la trontière franco-allemande, on note la pénétration de lotissements à l'intérieur des forêts, la création au milieu des bois de crassiers ou de décharges destinés à recevoir divers résidus industriels, 


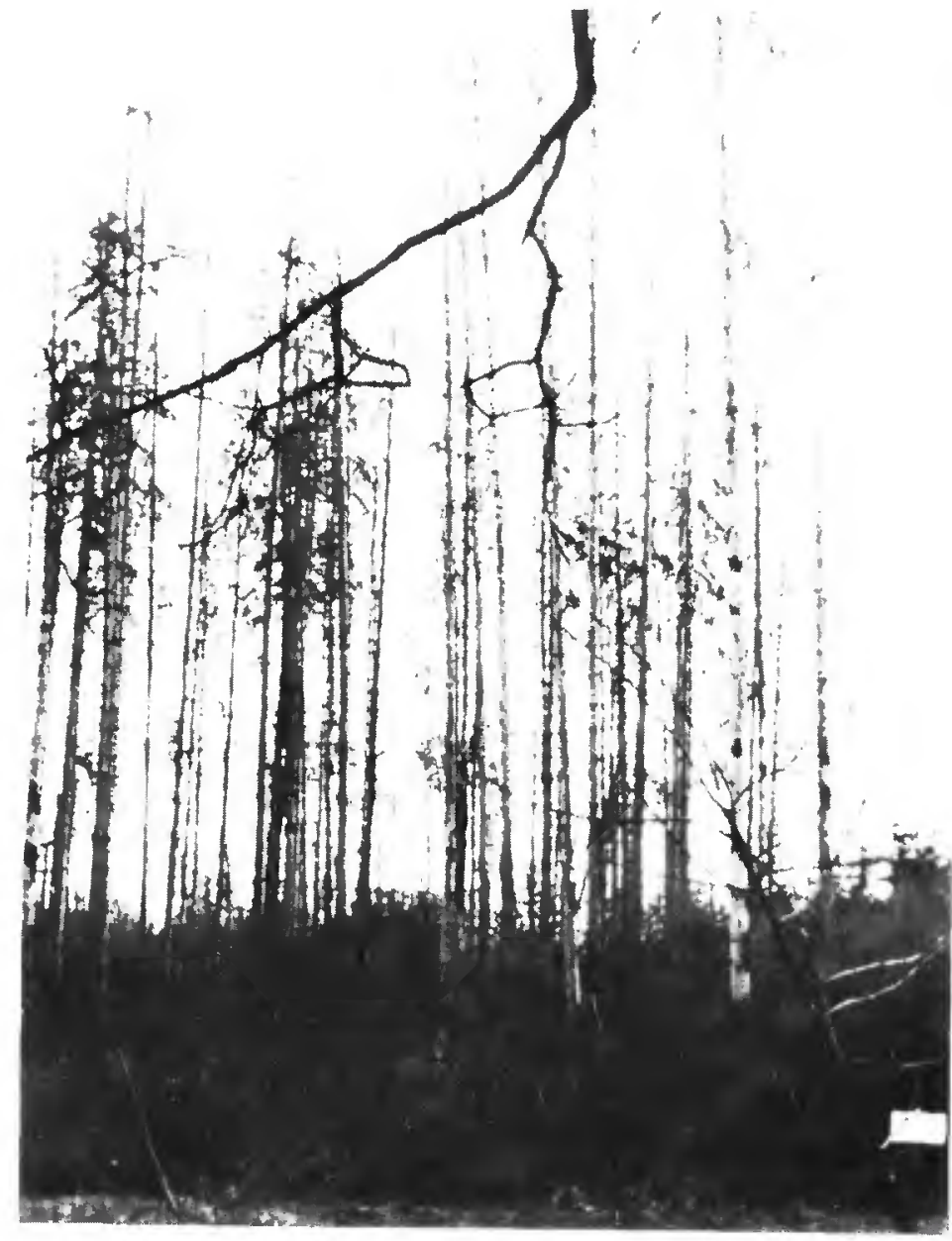

Bien que restes debout, les épiceas de la parcelle $n^{\circ} 151$ sont morts de pollution, comme l'in. dique l'écriteau en bas à droite. Photo BONNEFONT.

L'aménagement de la plate-forme du groupe VI de Carling a nécessité un important déboisement.

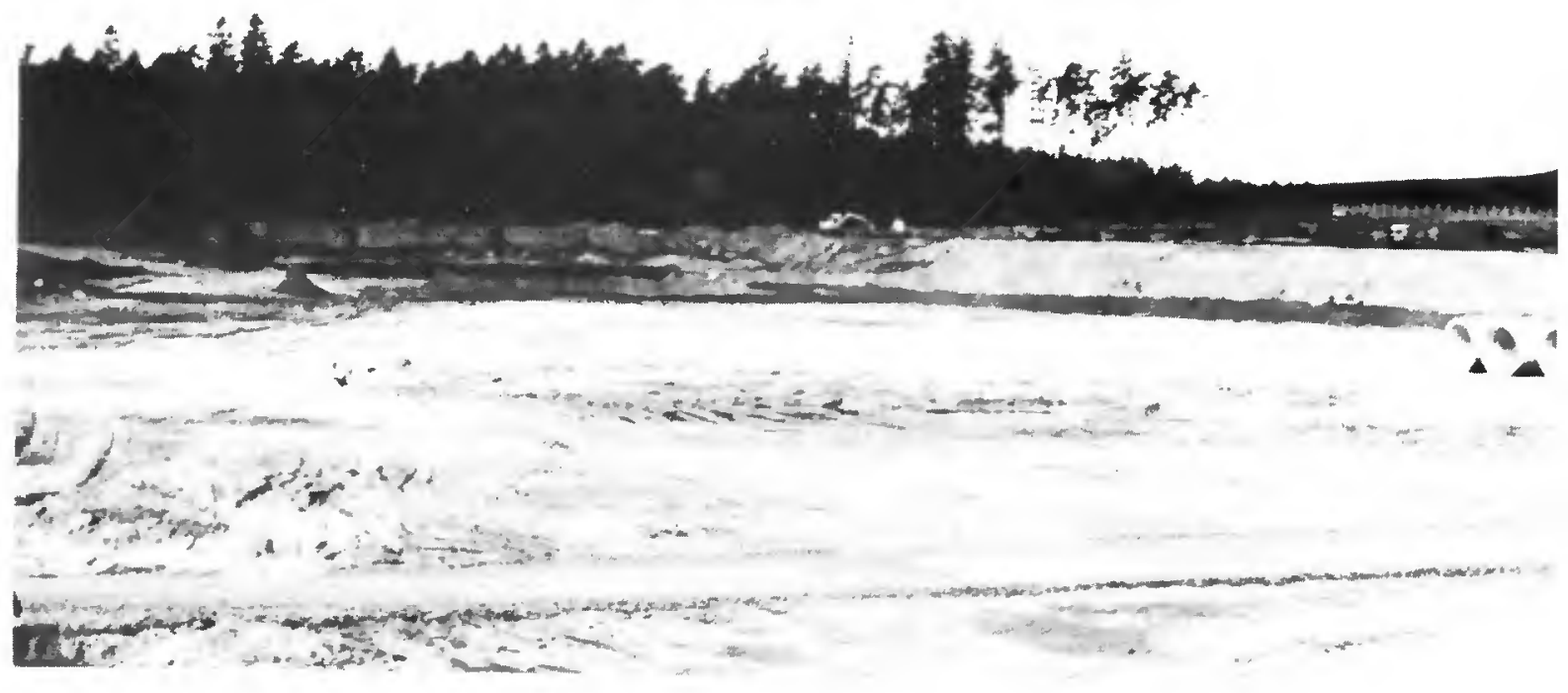



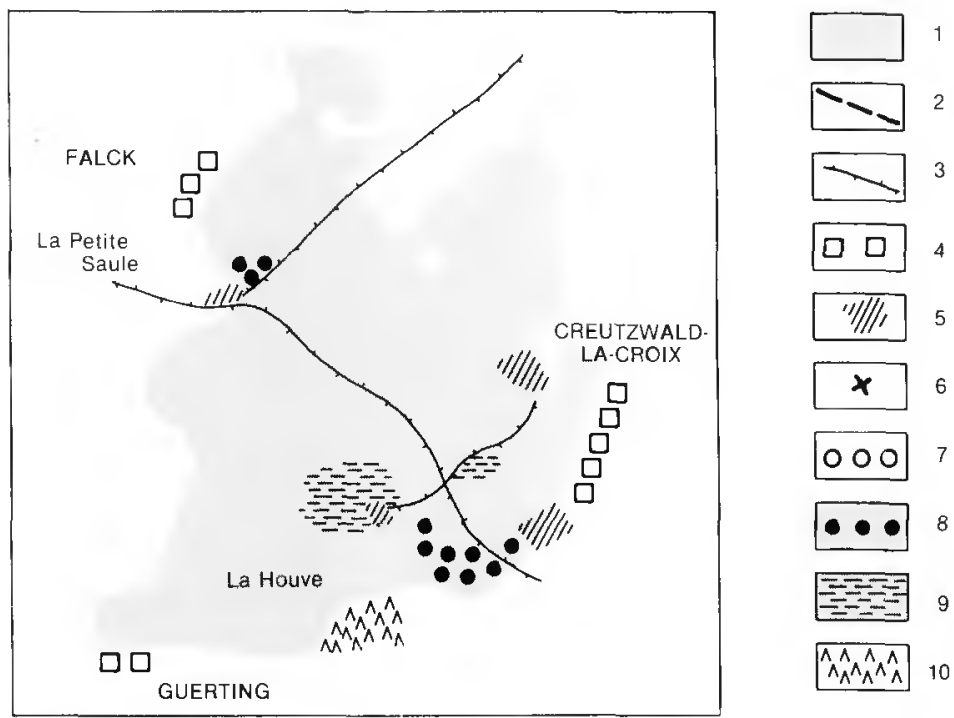

FORẼT DE LA HOUVE
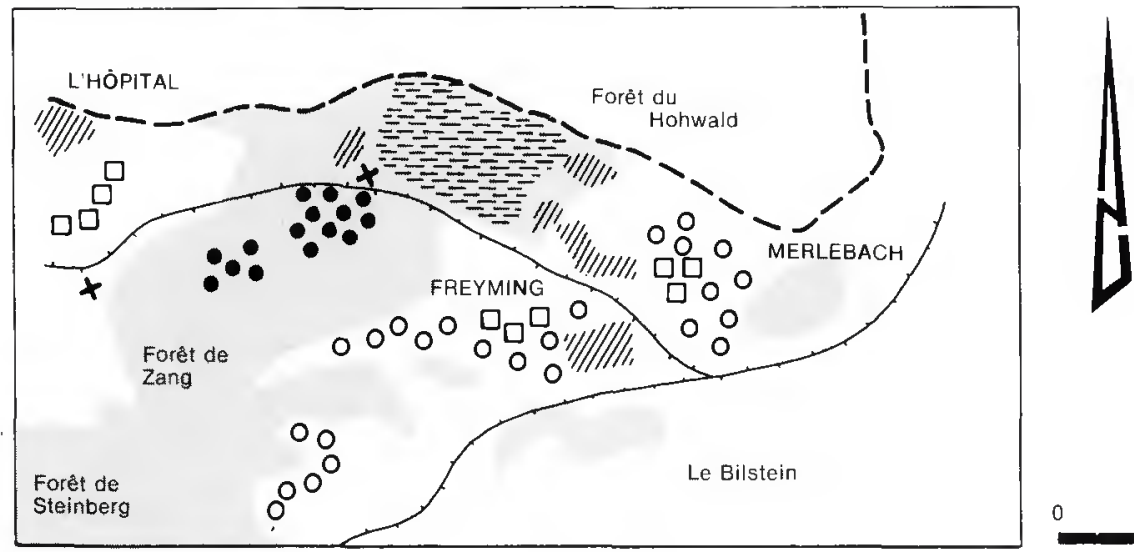

1 - forêt vers $1920 ; 2$ - frontiere franco-allemande ; 3 - ligne de chemin de fer ; 4 - ancien noyau villageois ; 5 - bâtiments industriels et cités vers $1920 ; 6$ - mine : 7 - extension nouvelle de l'habitat (hors de la forêt) : 8 - extension nouvelle de l'habitat (dans la forêt); 9 - ferrils, carrières, lagunes créés aux dépens de la forêt; 10 - reboisement. 
la formation d'étangs de lagunage pour la décantation des eaux polluées, l'ouverture de carrières de sable servant au remblaiement des galeries minières après le terme de leur exploitation. Outre la forêt de Saint-Avold, la forêt de La Houve et les bois qui entourent FreymingMerlebach présentent des exemples significatifs de toutes ces nuisances.

D'une façon générale, c'est dans le bassin houiller mosellan, resté plus longtemps en voie d'expansion, que les attaques subies récemment par la forêt ont été les plus spectaculaires. Elles ont été moins sensibles dans les régions sidérurgiques, où l'on n'a plus ouvert de nouvelle mine depuis près d'un demi-siècle et où les usines nouvelles, comme Sérémange et Gandrange n'ont pas été créees sur les plateaux boisés, mais dans les vallées. Cependant, les lotissements nouveaux fondés sur les plateaux au nom d'une conception nouvelle de l'habitat ont nécessité des déboisements plus ou moins importants. Le cas le plus significatif à cet égard est celui du village de Saint-Nicolas-en-Forêt.

Les études qui ont été à l'origine de la fondation du village (3) faisaient ressortir particulièrement les inconvénients des cités industrielles construites jusqu'ici dans la vallée de la Fensch : trop grande proximité des usines, insalubrité due aux fumées et à la mauvaise exposition, constructions ètablies sur des zones inondables, voisinage de routes à grand trafic. On désirait alors doter les nouvelles cités d'un environnement plus agréable et les séparer des usines par des "zones d'intervalle". C'est dans cette perspective que la SOLLAC a construit pour loger sa main-d'œuvre à partir de 1951 le nouveau village de Saint-Nicolas-en-Forêt. Situé comme son nom l'indique en pleine forêt, sur un terrain couvrant 50 ha appartenant aux communes de Fameck et de Ranguevaux, il a constitué une expérience d'urbanisme originale. D'autres réalisations du même type ont suivi, dont la dernière a été celle d'Homécourt-Haut.

Autour de l'habitat en clairière, le rôle joué par la forêt est à la fois récréatif et esthétique. Lieu de promenade, la forêt constitue aussi un écran visuel qui permet de séparer les maisons des bâtiments industriels. Dans un paysage de béton et d'acier, où l'homme se sent souvent déraciné, elle apporte une note naturelle et apaisante. Elle joue le rôle d'un filtre en interceptant les poussières émises par les usines. Elle forme enfin un écran acoustique qui atténue le bruit des installations industrielles.

Cependant, les lotissements réalisés en forêt présentent aussi beaucoup d'inconvénients, surtout pour la forêt elle-même. Introduire une population nombreuse dans un bois ou à sa bordure cause à la végétation un certain nombre de préjudices qui tiennent à une fréquentation trop intense et mal contrôlée: parmi les diverses nuisances, le piétinement qui tasse le sol et écrase les jeunes semis est sans doute la plus grave. II s'y ajoute dans certains cas des phénomènes d'assèchement du sol, lorsque les opérations de construction ont eu pour effet d'abaisser excessivement la nappe phréatique. A la longue enfin, il est évident que la multiplication des lotissements en forêt finirait par aller à l'encontre des avantages recherchés en faisant disparaître les massifs forestiers eux-mêmes. Pour toutes ces raisons, les nouveaux plans d'occupation des sols et les schémas de secteur établis en Lorraine mettent maintenant l'accent sur la nécessité de conserver intégralement toutes les parties boisées. Le Schéma d'aménagement de la Vallée de l'Orne-Amont, par exemple, exclut complètement toute idée de réalisation de nouveaux habitats au cœur des forêts $\left({ }^{4}\right)$.

(3) Comité d'Aménagement et du Plan d'Equipement de la Moselle. Fascicule 1/4/a, sans date : Aménagement du territoire en Moselle.

(4) Direction départementale de l'Equipement de Meurthe-et-Moselle. Schéma de secteur BRIEY-ORNE AMONT. Février 1973. 77 pages. 


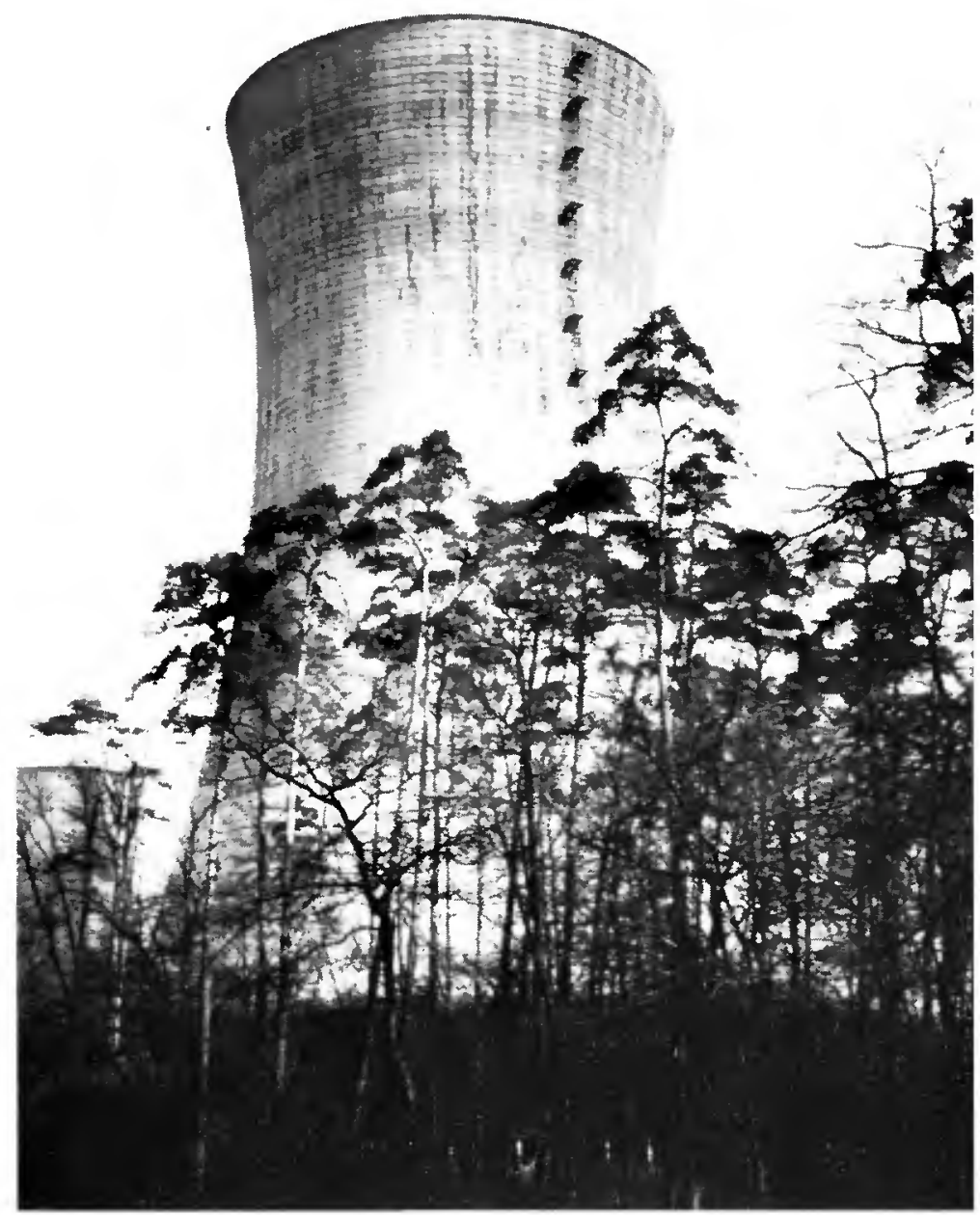

Auprès des gigantesques évaporateurs de Car-

Photo BONNEFONT ling, la forêt dé pins sylvestres est réduite à un simple écran. 
Nature, loisirs et torêt

\section{LA FORÊT ET L'ÉVOLUTION PRÉVISIBLE DES SITES INDUSTRIELS}

L'industrie lorraine est engagée depuis les années 1960 dans un long processus de reconversion, qui se traduit par le déclin de la sidérurgie et des mines, le développement d'industries nouvelles comme la chimie ou l'automobile, l'abandon de certains sites industriels anciens, la création de nouvelles zones industrielles.

La création de zones industrielles se fait parfois aux dépens d'une forêt. Chaque fois qu'elles atteignent une superficie importante d'un seul tenant, il existe de fortes chances pour qu'une parcelle boisée se trouve englobée dans le périmètre industriel ou au moins en limite de ce dernier. C'est ainsi que près de Toul la grande zone industrielle de Toul/Croix-de-Metz jouxte les bois de Villey-Saint-Etienne, qui sont maintenant devenus les victimes désignées d'une éventuelle future extension.

Le développement sur place des ensembles industriels existants constitue certainement la menace la plus grave portant sur les forêts. L'exemple de Carling nous a permis de l'illustrer parfaitement. Lorsqu'une installation industrielle a été réalisée à l'origine dans une clairière, ou à la lisière d'une forêt, et se trouve prise en tenaille entre les arbres et une zone d'habitation, les industriels disposent d'un moyen de pression considérable sur l'opinion et les pouvoirs publics pour obtenir l'extension de leur territoire aux dépens de la partie boisée. Leur argumentation a d'autant plus de chances de prévaloir que bien souvent la partie de la forêt qui se trouve directement au contact des usines a déjà été dégradée par l'ouverture de tranchées, l'installation de décharges ou l'effet de la pollution atmosphérique. On sacrifie alors assez facilement cette frange déjà abîmée sans vouloir songer que le front de l'attaque va simplement se déplacer un peu plus à l'intérieur du massif.

Inversement, l'abandon de nombreux sites industriels anciens devrait se traduire par une progression de la forêt. On a assisté ces dernières années en Lorraine à un glissement des activités sidérurgiques de la partie amont vers la partie aval des vallées. L'abandon des vieux sites industriels fait naître une sorte de "pollution visuelle" $\left({ }^{5}\right)$, surtout lorsqu'on se contente de récupérer les ferrailles et qu'on laisse à l'abandon les cheminées, les socles de hauts-fourneaux et les constructions en béton armé. Un reboisement judicieux pourrait permettre de réhabiliter ces sites. II en est de même des carrières qui, comme le fut celle de Merlebach, peuvent être reboisées en pins sylvestres à la fin de leur exploitation. Les crassiers enfin, Iorsqu'on ne désire pas les faire disparaître en les exploitant comme matériaux, peuvent aussi faire l'objet d'un reboisement, comme le montrent divers exemples, notamment à l'étranger (6). Cependant, il ne faut pas cacher que ces opérations de reboisement sont coûteuses, longues à réaliser, hasardeuses quant au résultat et généralement non rentables, car on est obligé d'utiliser des essences de faible valeur économique. Elles représenteront, si on les envisage, un coût élevé pour la collectivité dont il faut tenir compte dès maintenant dans les programmes d'aménagement.

Ainsi, on voit apparaitre actuellement en Lorraine deux évolutions divergentes, dont la simultanéité semble au moins à première vue peu rationnelle:

- d'un côté, on étend certaines plates-formes industrielles existantes en grignotant les forêts et en réalisant un maximum de concentration spatiale qui multiplie les nuisances pour l'environnement et même les risques pour la population. A Carling, par exemple, la limite de

(5) J. Jung. L'amélioration de l'environnement dans une région d'industrie lourde. Sciences pharmaceutiques et biologiques. IVe Colloque National d'Hygiène de l'Environnement et des Collectivités locales, Nancy, octobre 1975, pages 55-58.

(6) Tristan Pauly. Reboisement de terrils en Allemagne Fédèrale. Revue forestière frança/se, $n^{\circ}$ 6, nov.-déc. 1971. pages $629-635$. 
saturation paraît bien près d'être atteinte et les protestations d'écologistes et d'usagers prennent de plus en plus d'ampleur;

- d'un autre côté, on abandonne des sites industriels anciens, auprès desquels la population est pourtant solidement implantée. Si on désire les "réhabiliter " complètement, leur reboisement sera long, coûteux et peu économique.

Dans ces conditions, on se demande s'il ne vaudrait pas mieux, pour l'équilibre de la population comme pour une bonne gestion de la forêt, faire l'effort de réutiliser en priorité pour l'industrie les sites industriels existants. Certes, certains sites anciens peuvent souffrir d'une insuffisante "accessibilité"; cette politique exigerait sans doute des pouvoirs publics un aménagement plus complet des voies de communication. Mais la question vaut d'être étudiée. A une époque où l'humanité prend conscience que toutes ses ressources sont limitées, ne faut-il pas songer à combattre d'abord tous les gaspillages d'espace, en laissant aux sols boisés leur vocation forestière et aux terrains industriels leur vocation industrielle?

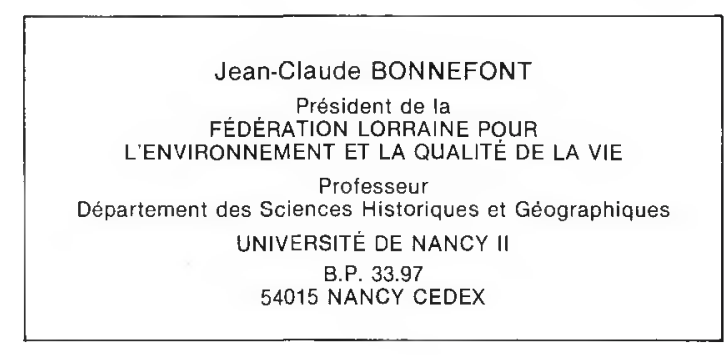

